\title{
Value-Driven Robotic Digital Twins in Cyber-Physical Applications
}

This paper was downloaded from TechRxiv (https://www.techrxiv.org).

\section{LICENSE}

CC BY-NC-SA 4.0

SUBMISSION DATE / POSTED DATE

$17-01-2021 / 19-01-2021$

CITATION

Guiffo Kaigom, Eric; Rossmann, Juergen (2021): Value-Driven Robotic Digital Twins in Cyber-Physical Applications. TechRxiv. Preprint. https://doi.org/10.36227/techrxiv.13600964.v1

$\mathrm{DOI}$

10.36227/techrxiv.13600964.v1 
(C) 2020 IEEE. Personal use of this material is permitted. Permission from IEEE must be obtained for all other uses, in any current or future media, including reprinting/republishing this material for advertising or promotional purposes, creating new collective works, for resale or redistribution to servers or lists, or reuse of any copyrighted component of this work in other works.

Value-Driven Robotic Digital Twins in Cyber-Physical Applications

Eric Guiffo Kaigom and Jürgen Roßmann,

IEEE Transactions on Industrial Informatics (2020),

https://doi.org/10.1109/TII.2020.3011062

\section{BibTeX:}

@article $\{$ kaigom2020value,

title $=\{$ Value-Driven Robotic Digital Twins in Cyber-Physical Applications $\}$, author $=\{$ Kaigom, Eric Guiffo and Rossmann, Jurgen $\}$,

journal $=\{$ IEEE Transactions on Industrial Informatics $\}$,

year $=\{2020\}$,

pages $=\{1-11\}$,

publisher $=\{$ IEEE, New York, NY $\}$,

note $=\{$ Print ISSN: 1551-3203 Electronic ISSN: 1941-0050 $\}$,

doi $=\{$ https://doi.org/10.1109/TII.2020.3011062 $\}$,

keywords $=$ \{Digital twins, robotics, cyber-physical-systems, industry 4.0, simulation, human-robot-interaction $\}$, url $=\{$ https://ieeexplore.ieee.org/stamp/stamp.jsp?tp=\&arnumber $=9145799\}\}$ 


\title{
Value-Driven Robotic Digital Twins in Cyber-Physical Applications
}

\author{
Eric Guiffo Kaigom, Jürgen Roßmann
}

\begin{abstract}
Although the skills of robot manipulators are becoming technically more complex, the unprecedented costeffective access to recently unveiled intelligent robots has the potential to unleash as yet unimagined automation capabilities. A key technology behind this opportunity for companies to gain a competitive edge through an informed and intelligent robotized automation is the robotic digital twin (RDT). As such, the RDT will be instrumental in mirroring targeted properties of a physical robot to obtain a digital sibling flexibly harnessed in virtual testbeds to understand, predict, and shape the robot performance. However, these objectives remain challenging to well-established simulators. This is because the architectural and functional capabilities they support are not sufficiently in-line with ever-growing and varying demands for agile and cost-efficient manipulations. As a consequence, robot stakeholders can hardly use RDTs to unlock opportunities and meet needs from prospective markets. This paper contributes to addressing this gap. We introduce a novel concept for the development of a RDT that helps create and add value to current and future robotized cyber-physical applications. Hereinafter referred to as the value-driven RDT (vdRDT), it systematically captures the robot dynamics and purposefully farms data, about which its services reason, to facilitate insight and deliver capabilities as well as benefits to stakeholders. Experiment results show that vdRDTs enlarge the scope of, adapt to, and revitalize robotized applications carried out in different fields.
\end{abstract}

Index Terms-Digital twins, robotics, cyber-physical systems

\section{INTRODUCTION}

A lthough the COVID-19 lockdown has affected industries, retooled robots have been crucial for manufacturing in at least two aspects: making production lines less dependent on collaborators to meet social distancing rules and continuously producing new products to fight the coronavirus [1]. In the midst of this pandemic and beyond, the vital role of robots has been emphasized by a predicted compound annual growth rate of their market size of $10.4 \%$ during the next five years [2].

Enterprises can take advantage of this growth momentum, digitalization, and knowledge transfer to reinvent and reinvigorate their automation capabilities. Meaningful information

Parts of this work were carried out within the scope of the project IBOSS-3 supported by the German Aerospace Center with funds of the German Federal Ministry of Economics and Technology (BMWi) under grant agreement No 50 RA 1203, the European Project ReconCell supported by funds from the Horizon 2020 Research and Innovation Programme under grant agreement No 680431, and the project ProDemo-2 supported by the German Federal Ministry of Economics and Technology (BMWi) under the grant agreement ZBG 09488/08.

Eric Guiffo Kaigom (corresponding author) was with the institute for Man-Machine-Interaction, Faculty of Electrical Engineering and Information Technology, RWTH Aachen University, Ahornstrasse 55, Aachen, Germany (e-mail: eric.kaigom@gmx.net).

Jürgen Roßmann is with the institute for Man-Machine-Interaction, Faculty of Electrical Engineering and Information Technology, RWTH Aachen University, Ahornstrasse 55, Aachen, Germany (e-mail: rossmann@mmi.rwthaachen.de).

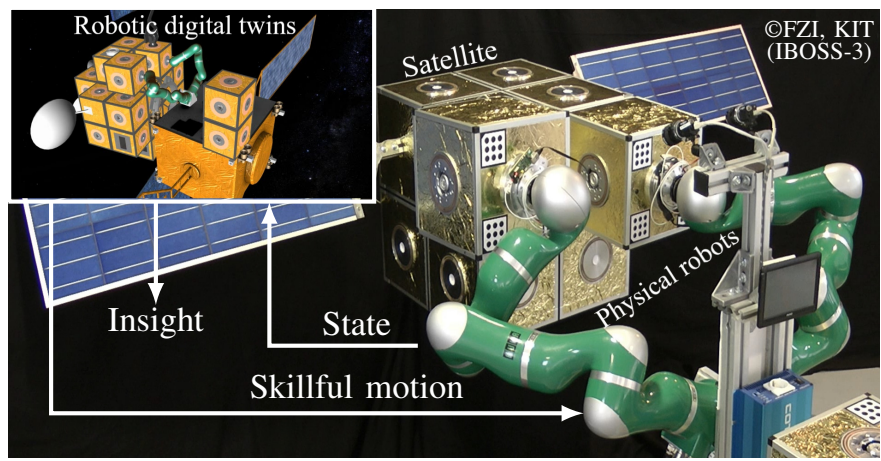

Fig. 1: Robotic digital twins of physical service robots as enablers for a cost-efficient analysis of satellite servicing.

unveiled from process and robot data can be used to holistically predict skillful and economic joint motions that add value across business units. Also, well-informed and timely decisions leading to adaptive manipulations help transform changing market opportunities into stable sources of profit. To meet current and upcoming pressing needs for smart and transparent automation, enterprises therefore seek intelligent, flexible, and scalable solutions that broaden situation awareness along short and long term strategies. Expectations placed on such software include three goals. Firstly, delivering motions that reduce manipulation costs. Secondly, safely and competitively adapting manipulations to uncertainties. Finally, turning skillful motions into outcomes that align with expectations of stakeholders.

Within the realm of digitalization efforts currently being made to meet these goals, the digital twin (DT) is playing a pivotal role. A DT is a computer-executable suite of integrated models, data, and algorithms that capture and render specific characteristics of an existing physical asset. As far as a robot is concerned (see Fig. 1), its virtual replica with respect to targeted proprieties is the robotic DT (RDT). A key characteristic exhibited by a RDT is the robot dynamics which, when accurately simulated, is highly informative from technical and economic viewpoints. Still, it is worth noting that, beyond the standard robot simulation, the RDT is tightly coupled with its physical counterpart [3]. Status data, such as joint velocities and torques, are measured from the robot, applied in real-time to the RDT through network coupling, and managed in e.g. an in-memory database to "continuously update the DT with all the evolutionary changes and modifications in the physical system" [3]. The RDT can scrutinize these historical data to assess safety, cycle time, and operational costs, as well as detect anomalies. Gained insights impact decisions on how efficiently 


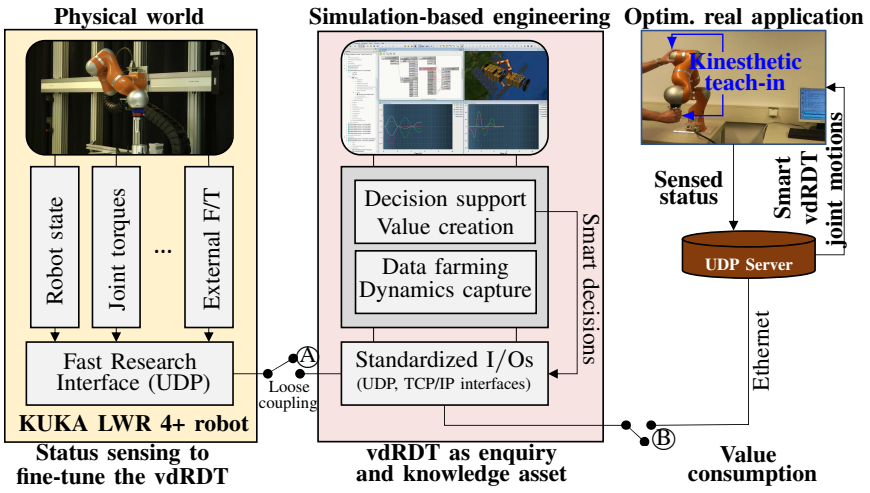

Fig. 2: Fine-tuning (1.h.s) and employing (r.h.s) the vdRDT.

and profitably robotized tasks have been carried out thus far.

However, improving the technical and economic viability of industrial applications often builds upon joint motions completely different from historical data in the RDT. Highmix-low-volume manufacturing is an application example in which volatile demands require agile, investigative, and expository capabilities from a RDT. These include projecting the robotized accommodation of uncertainties (e.g., external forces) onto models of upcoming customer orders, revealing properties that emerge from ongoing tasks, and creating value. But, because projection results usually culminate in a geometrical abstraction and simulation of robotized tasks, current RDTs fail to generate substantial value. Such a shortcoming differently manifests itself when the RDT cannot reflect the internal robot dynamics or even deliver intelligent manipulations constrained by physical interactions with humans or work-pieces to complete a task, as is the case in practice. In light of this gap, RDTs are up to now hardly helpful in harvesting and sharing information to anticipate, adapt, and quickly react to changing market trends and needs through actionable and competitive insights into robotized processes.

\section{CONTRibutions}

We describe the development of the value-driven RDT (vdRDT) that consumes real and synthesized data to provide applications with information useful for shaping and enhancing robot performances while empowering stakeholders in decision-making. A vdRDT not only mirrors the historic and current robot behavior, but matures to operate beyond the motion experience of the robot, thus expanding the prediction and value creation horizon of a standard RDT (see middle of Fig. 2). This is done by exploiting a persistent capture of the robot dynamics. For desired robot states (i.e., joint positions and velocities), a vdRDT farms (i.e., grows) and harnesses data in simulation to uncover motions adding value to applications. We adapt the architecture for programming and controlling industrial robots from [4], and extend it in three core aspects that transform vdRDTs into drivers for building a knowledge asset for stakeholders (see Fig. 3). This usefulness is achieved by:

- rendering customizable dynamics of free-space and perturbed robot motions in virtual environments. The mature $\mathrm{vdRDT}$ is then ready to support prospective applications.

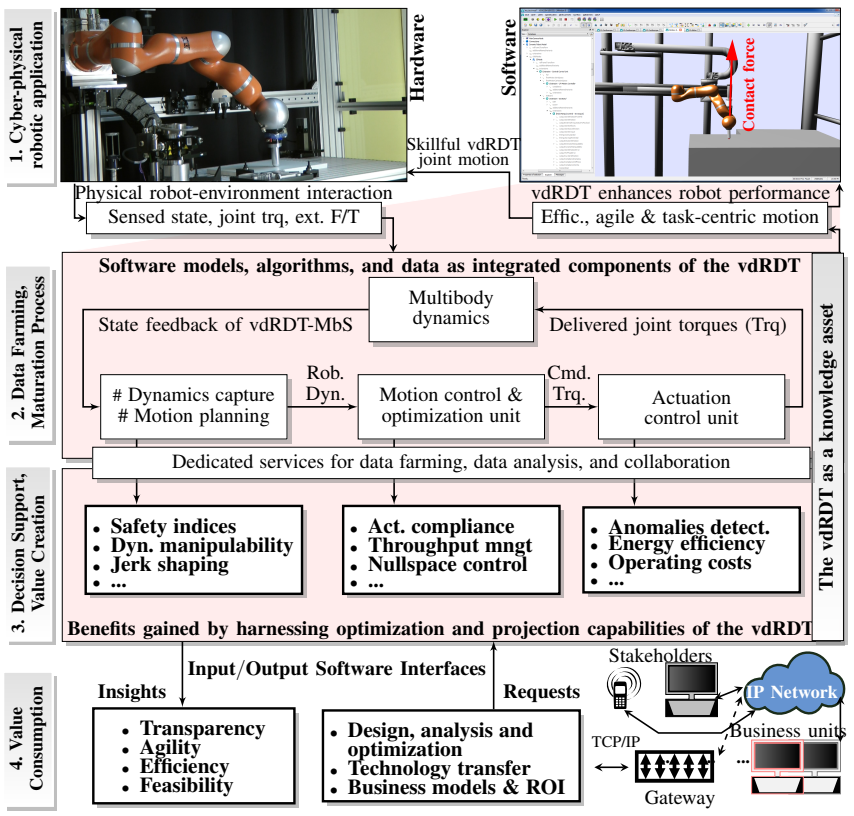

Fig. 3: A vdRDT shapes the robot performance and transforms data into competitive information that empower stakeholders.

- equipping the vdRDT with active compliance. This enlarges the range of applications supported by RDTs.

- enriching the vdRDT with services that cultivate, analyze, and share data to help stakeholders identify advantages, make well-informed decisions faster, and obtain appropriate performances for a gainful usage of robot assets.

In addition to mirroring the past and current robot behavior, a vdRDT helps enterprises quickly project robotics capabilities and ascertain prerequisites for task completion. An example is the proactive reduction of the robot energy consumption in future applications. This consumption is a function of envisaged joint velocities tracked by the vdRDT and delivered joint torques. Motion dynamics captured by the vdRDT provide these torques even when the vdRDT is fully decoupled from the robot. As depicted in Fig. 2, after dynamics capture, during which the switch (A) is closed, a vdRDT operates independently of the robot (A) open) to identify advantages and strengthen application performances (B) closed). The decoupled employment of the vdRDT as a reliable surrogate is useful when e.g. the robot is not available for what-if experiments, operational conditions of the robot are different from those of its vdRDT, or single contributions that sum up to joint torques are required for performance shaping. Insights gained from a vdRDT ultimately enrich current and prospective applications, as shown in Figs. 2 and 3. For example, services run by the vdRDT can scale joint velocities or optimize redundant trajectory coefficients to deliver joint motions that minimize the consumed energy. As the robot is actuated to track these synthesized joint trajectories in real applications, its vdRDT has already created value, since engendered energy supply costs are considerably cut down.

A vdRDT offers interfaces to users for specifying both technical and economical objectives, and exposes relevant results of their fulfillment (see layers 3 and 4 in Fig. 3). Specifically, 


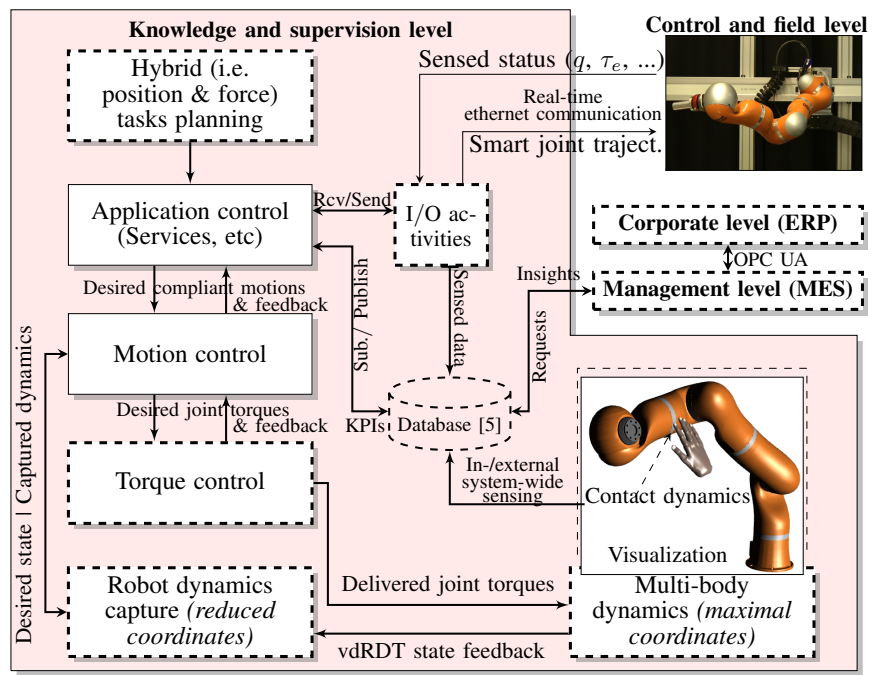

Fig. 4: vdRDT architecture, as part of computer-integrated manufacturing. This architecture and its integration adapt and extend [4]. Added functionalities are in dashed boxes.

it provides its connected ecosystem with cross-disciplinary incentives and leverage points related to the robot usage and performance. Business units oversee robotized productions throughout the organization via an enterprise resource planning (ERP). Units take advantage of these shared levers to jointly contribute to the overall enterprise prosperity (see Fig. 4). They do that by using vdRDT insights to increase the profitability of robotics on which they focus from different viewpoints.

The remainder of the paper is structured as follows. Section III presents related recent works. Section IV describes the maturation process of the vdRDT. Section V addresses value creation. Section VI describes conducted experiments. Section VII discusses the paper and section VIII concludes it.

\section{STATE OF THE ART}

Few efforts have been recently devoted to the use of RDTs to enhance the outcomes of robotized industrial and service applications. In [6], a RDT simulated in Visual Components monitors a robot by collecting data, such as velocity, torque, and temperature, sensed from joint motors. Conversely, the RDT modifies the robot posture by specifying joint positions. A similar bidirectional loop is proposed in [3], [7], where the RDT is, in addition, associated with a digital human model. Both DTs are kinematically run in Tecnomatix to enhance workflows in manual assembly based upon physical humanrobot-interaction (pHRI). RDTs and virtual reality (VR) are combined in [8] to remotely operate a Universal Robots (UR) $10 \mathrm{arm}$. Similarly, a UR $3 \mathrm{arm}$ is controlled in [9] for remote surgery. Service- and event-driven intelligent solutions are developed in [10] to highlight that technologies based upon Industry 4.0 (I4.0) meet needs in the Circular Economy. For that, CIROS $^{\circledR}$ is utilized to reconfigure robots and launch orders by using the built-in manufacturing execution system (MES). In [11], RDTs are operated in Plant Simulation to evaluate benefits of using collaborative robots, such as KUKA LWR IIWA, in I4.0-based automotive production lines. Insights are provided to operators in [12] to carry out remote processes involving $U R 5$ arms in I4.0 applications. To this end, RDTs are run in a simulation environment made up of ROS and Unity $3 D$.

Though approaches mentioned in this section draw upon 3D simulation, popular and comprehensive simulators are missing the systematic capture of the robot dynamics. Furthermore, skillful capabilities endowed in recently unveiled collaborative robots are not considered. A salient example is the lack of a beneficial handling of uncertain external wrenches exerted on the robot, which is prevalent in e.g. pHRI and cellular manufacturing. This gap, addressed in section IV, has important ramifications and implications for both contemporary and upcoming applications. Indeed, as pointed out in experiments presented in section VI, besides crucial safety concerns, compliant motions considerably influence cycle time [13], consistency of product quality [14], and energy consumption [15].

Notice that approaches mentioned in this section illustrate the potential of RDTs to minimize production costs, enhance product quality, and enable well-informed decisions in lean manufacturing. Nevertheless, they also reveal challenges faced by developing RDTs for these purposes. Since these contributions build on a kinematic abstraction of robots, they are relevant for applications in which RDTs do not exchange mechanical energy with the environment. Obviously, this restricts the range of possible applications and value creation. In fact, considerable contact dynamics are omnipresent in production processes based upon pHRI, and some skillful joint velocities are dynamics-dependent in the space environment (see section $\mathrm{V}-\mathrm{C}$ ). Furthermore, from the viewpoint of dynamics and control, using a digital model to approximate the interactive human behavior is a challenging undertaking. This is because, for instance, the human endpoint stiffness depends on muscle co-contractions and arm posture [16]. By contrast, contact forces measured in pHRI are used in sections VI-C and VI-E as real-time inputs to increase the usefulness of vdRDTs and significance of insights gained. Also, as the coupling between the RDT and robot is removed, the RDT is no longer aware of the robot dynamics for joint motions that differ from the recorded history of trajectories and external torques. This shortcoming compromises the maturation process of the RDT.

\section{MATURITY}

In [17], how the concept of DT connects with maturity, in general, was recently described as the "level of sophistication" of the DT. In our particular robotics context, we aim at making a mature vdRDT capable of accurately rendering joint torques of its paired robot under similar external disturbance forces.

\section{A. Robot dynamics capture}

Without experiencing external disturbances, a robot exhibits its nominal dynamics. As it exchanges mechanical energy with its environment, the robot is in the interactive dynamics mode. Interactions include undesired collisions and intentional contacts, such as during manual guidance. To recreate both nominal and interactive dynamics in 3D simulation, two modeling schemes for multi-body systems were developed from scratch in $\mathrm{C}++$, integrated, and customized in this work. The 
first scheme offers an intuitive modeling of the vdRDT and gives access to it as a component of a set of multi-body systems, possibly in physical interaction. The other scheme modularly isolates single torque-related terms that together contribute to the overall robot dynamics. It thereby lays the ground for a torque-based robot control that bridges both schemes, as depicted in Fig. 4. Finally, dynamics parameters are fine-tuned to bring joint torques closer to measured values.

1) Virtual world modeling in maximal coordinates: The physics-based environment of vdRDTs is exemplified on the lower r.h.s of Fig. 4. Free-space motions and physical interactions between multi-body systems are modeled in maximal (i.e., Cartesian) coordinates and rendered by integrating

$$
M_{m c} \dot{\nu}_{m c}=f_{m c}+f_{m c_{c}}
$$

to solve for $\nu_{m c} \in \mathbb{R}^{6 n_{B}}$. $\nu_{m c}$ contains linear and angular velocities of all the $n_{B}$ bodies of systems like vdRDTs and workpieces. $M_{m c} \in \mathbb{R}^{6 n_{B} \times 6 n_{B}}$ is a block diagonal matrix with $i$-th block $W_{m c}^{i, i \in\left\{1, \cdots, n_{B}\right\}}=\left[\begin{array}{cc}m_{i} U_{3} & 0_{3} \\ 0_{3} & I_{i}\end{array}\right]$, where $m_{i}$ and $I_{i}$ are the mass and inertial tensor of the $i$-th body. $U_{3}$ and $0_{3}$ are the unit and zero matrices of $\mathbb{R}^{3 \times 3} \cdot f_{m c}$ contains external, as well as angular velocity-dependent forces acting on bodies. $f_{m c_{c}}$ denotes forces ensuring constraints. Specifically, relative velocities are intuitively enforced between pairs of bodies to assemble multi-body systems, such as a vdRDT, by using $f_{m c_{c}}$. For instance, a rotatory joint that articulates a body pair is enabled by using $f_{m c_{c}}$ to forbid relative motions in the three translational and two rotatory dimensions. Notice that $f_{m c_{c}}$ fulfills this goal without injecting or extracting energy from the multi-body system, since it acts orthogonally to the direction of allowed motions. Soft contacts are exhibited and customized through spring-damper models with relaxed interpenetration constraints, as detailed in [18]. In equation (1), $f_{m c_{c}}$ is obtained in our implementation from the automated construction on-the-fly of a Jacobian for each constraint type, and by solving an iterative complementary problem for the magnitude of impulses so that $f_{m c_{c}}$ meets constraints [18], [19]. Our implementation supports the projected Gauss-Seidel (used in this work) as well as Dantzig solvers. Though maximal coordinates modeling offers a generic and systematic assembly of a tree-like multi-body system, it nonetheless involves more degrees of freedom (DoF) than needed to control a vdRDT.

2) Nominal robot dynamics in reduced coordinates: The dynamics of a vdRDT is expressed on the body side as [20]

$$
\left\{\begin{array}{l}
M(q) \ddot{q}+c(q, \dot{q})+g(q)=\tau_{J}+\tau_{e} \\
B \ddot{\theta}+\tau_{J}=\tau_{m}-\tau_{f} \\
K(\theta-q)=\tau_{J}
\end{array}\right.
$$

Vectors $q$ and $\theta$ of $\mathbb{R}^{n}$ contain joint and gear-reflected motor positions. $M=J_{m c}^{T} M_{m c}^{*} J_{m c} \in \mathbb{R}^{n \times n}$ is the mass matrix. $M_{m c}^{*}$ is built similarly to $M_{m c}$ in section IV-A1, however, using only the $n$ articulated bodies of the vdRDT with linear and angular velocities $v_{i}$ and $w_{i}, i \in\{1, \ldots, n\}$. Observe that $n<6 n_{B}$, even when the environment is made up of the vdRDT only. The Jacobian $J_{m c}$ maps $\dot{q}$ to $\nu_{a}=$ $\left[v_{1}^{T}, w_{1}^{T}, \ldots, v_{n}^{T}, w_{n}^{T}\right]^{T}$. A factorization $c(q, \dot{q})=C(q, \dot{q}) \dot{q}$ of the Coriolis/centrifugal matrix $C$, which preserves the skew-symmetry of $\dot{M}-2 C$ for motion control purposes, is given in [21]. $g$ denotes gravitational torques obtained by projecting the vector $F_{g}=\left[\delta_{1}^{T}, \ldots, \delta_{n}^{T}\right]^{T}$ of gravity forces $\delta_{i}=\left[0,0,-9.81 * m_{i}, 0,0,0\right]^{T}$ for each body onto $J_{m c}^{T} \cdot \tau_{J}$, $\tau_{m}, \tau_{f}$, and $\tau_{e}$ are joint, motor, friction (e.g., Coulomb, Lugre), and external torques. Diagonal matrices $K$ and $B$ contain structural joint stiffnesses and gear-reflected motor inertias.

In practice, the robot is sometimes upgraded. A force/torque sensor may be mounted on the end-effector to measure external wrenches. To take such extensions into account, the Steiner's theorem is used to build an equivalent composite body by using inertia properties of the sensor and end-link. Similar modifications of the robot are logged and memorized by the evolving vdRDT for the traceability of its previous configurations.

3) Free-space motion and physical interaction control:

Agile manipulations are enabled by endowing the vdRDT with compliant motion tracking. As wrenches act on the vdRDT, it absorbs the impact energy and renders a desired stiffness at its steady state in joint or Cartesian space. Furthermore, the apparent inertia of the vdRDT can be customized to shape its acceleration. For these purposes, a second order massdamper-spring relationship is established between external disturbances and the compliant deviation in both spaces.

In the Cartesian space, the impedance relationship reads [22]

$$
\left\{\begin{array}{l}
M_{C_{T}} \Delta \ddot{x}_{d c}+D_{C_{T}} \Delta \dot{x}_{d c}+S_{C_{T}} \Delta x_{d c}=f_{e_{T}} \\
M_{C_{R}} \Delta \dot{w}_{d c}+D_{C_{R}} \Delta w_{d c}+K_{C} \epsilon_{d c}=f_{e_{R}}
\end{array}\right.
$$

In the translational part given by equation (3a), $\Delta x_{d c}=x_{d}-$ $x_{c} \in \mathbb{R}^{3}$ is the difference between desired and compliant positions of the end-effector, and $f_{e_{T}}$ is the force acting on it. Diagonal matrices $M_{C_{T}}, D_{C_{T}}$, and $S_{C_{T}}$ of $\mathbb{R}^{3 \times 3}$ contain desired inertia, damping, and stiffness values. Rotational motions in equation ( $3 b)$ are free of representation singularities, since the vector part $\epsilon_{d c} \in \mathbb{R}^{3}$ of the unit quaternion error between desired and compliant frames describes the compliant deviation. Hence, the vdRDT carries out tasks without restriction in the orientation of its end-effector. The matrix $K_{C}$ is built such that the external elastic moment $f_{e_{R}}$ and relative orientation of both frames are geometrically consistent [22]. vdRDTs take advantage of this property to facilitate the planning of rotational compliance about eigenvectors of the desired stiffness matrix. Assembly and docking are application examples with relevant relative motions. $\Delta w_{d c}$ is the deviation between desired and compliant angular velocities. $M_{C_{R}}$ and $D_{C_{T}}$ contain desired rotational inertias and stiffnesses. With $\ddot{P}_{c}=$ $\left[\ddot{x}_{c}^{T}, \dot{w}_{c}^{T}\right]^{T}$ as desired compliant acceleration from equations (3a) and (3b), desired compliant joint accelerations follow as $\ddot{q}_{c}=\bar{J}\left(\ddot{P}_{c}-\dot{J} \dot{q}\right)+\eta \xi$. Herein, $\bar{J}=M^{-1} J^{T}\left(J M^{-1} J^{T}\right)^{-1}$ and $\eta=U_{n}-\overline{J J}$, where $J$ is the end-effector Jacobian. The choice of $\bar{J}$ to construct the nullspace $\eta$ of $J$ allows the vdRDT to decouple the enforcement of a secondary goal (see Figs. 5(a)-5(c)), which has the arbitrary cost-function gradient $\xi$, from the Cartesian motion at the acceleration stage. For full-body compliance based upon joint admittance control shown in Fig. 5(d), external joint torques are used to compute desired compliant joint accelerations from a relationship similar to equation (3a). Compliant joint trajectories in both control schemes are tracked by the motion control module in 


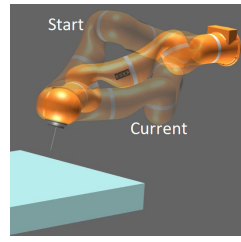

(a) Self motions.

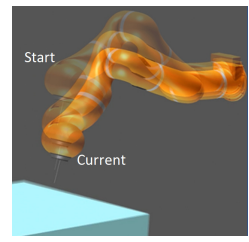

(b) Stabilized nullspace. (c) Active Cart. impedance.

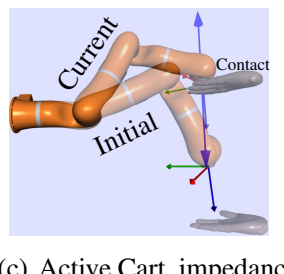

End: contactless, initial posture

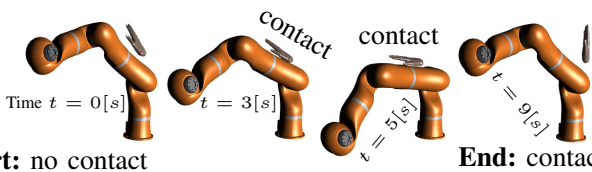

Start: no contact

(d) Externally disturbed vdRDT endowed with active joint admittance.

Fig. 5: Top: Nullspace-stabilized Cartesian compliance control of a 7 DoF vdRDT. Down: Full-body compliance control.

Fig. 4 by using computed-torque that cancels joint couplings or robust passivity-based methods. In the later case, $c(\dot{q}, q)$ is factorized following section IV-A2. As disturbances vanish, compliant motions converge to the desired ones, as shown in Fig. 5(d). Thus, a vdRDT autonomously and smoothly changes over between accurate trajectory tracking and accommodating external uncertain wrenches without control switch. This aligns with the autonomy expected in the 4. DT-level in [17].

4) Tuning dynamics parameters: Robot dynamics capture results from optimizing dynamics parameters to bring joint torques of the vdRDT closer to those of the robot. Since $\tau_{J}$ is sensed by most robots, such as KUKA LWR III, 4+ and IIWA, as mentioned in [20], optimized dynamics parameters are those involved in equation (2a). Additional drive train parameters are obtained from the literature, such as from [23] for joint stiffnesses in our KUKA KR 16 model in Fig. 6(b) and [20] for the gear-reduction range in our LWR /IIWA models. Feasible default parameters are used to initialize the virtual robot model of the vdRDT during its offline modeling, as in [24], [25]. Feasibility refers to e.g. a positive mass and suitably bounded link inertias. As simulations run, our extended implementation of the Particle Swarm Optimization (PSO) [26] continuously finetunes these parameters for maturation. Basically, the PSO displaces a set of optimization vectors called particles in a search area, seeking particle positions at which the cost-function is optimized. This process is repeated while adjusting the previous velocity to obtain the next position of each particle until the cost-function is lower than a defined threshold. After a maximal number of $N_{P}$ rounds, where $N_{P}=500$ by default, the optimization is stopped. Our implementation additionally tracks the convergence rate of the cost-function to detect a possible stagnation, in which case the swarm is reconfigured around single personal best positions. For trajectories tracked by the robot and its vdRDT, the sum of the squared difference between measured and simulated joint torques at discrete time steps is the cost-function. During the optimization, the vdRDT is coupled with the robot to gather measured joint torques (see 1.h.s of Fig. 2). One advantage of PSO is its affinity for threadbased parallelization on GPU [27]. This makes it possible to speed up the maturation process as a basis for value creation.

\section{VAlue Generation}

Services of mature vdRDTs identify advantages and highlight shortcomings in current and future cyber-physical applications (see Fig. 3). Achieving this goal rests on three pillars: first, data growing services (DGS) exploit captured dynamics and robot properties to farm data with focus and transparency. Second, data analytics services (DAS) scrutinize these data and optimize cost-functions to uncover efficient vdRDT motions. Third, data transfer services (DTS) forward these motions to applications to support decisions and materialize value added.

\section{A. Service-Orientation}

Ensuring visibility and efficiency in robotized production operations is an objective of the MES in Fig. 4. To gain insight and leverage processes, the MES first provides the vdRDT with information about scheduled tasks. These include desired joint states as well as indexes like dynamic manipulability to evaluate how joint torques map into Cartesian accelerations. Then, the MES requests crucial information, such as motion feasibility, from the vdRDT. Requests are processed by self-contained services supervised by the application control. Depending on the context deducted from parameters passed by requests, the application control executes the appropriate service. Information is returned according to the predefined level of detail or user experience. Services exchange results via standardized interfaces that also support internet sockets. Thus, services are interconnected even remotely together to deliver new functionalities, manage changes, and propagate dashboard information missed from proprietary robots via the MES to raise awareness in processes, performances, and support decisions. The information includes predicted throughput under compliance, energy-based safety metrics, and cross-domain joint motions that meet distinct needs of business units integrated via the ERP. How the Cartesian compliant behavior of a redundant robot impacts cycle time is, for instance, combined with minimizing, through posture control, the mass felt at the end-effector. Such optimizations are achieved by services that cascade gradients of cost-functions from business units in successive nullspaces [28]. Multiple vdRDTs can be spawn (see Fig. 14(b)) for free to intelligibly visualize condensed complex information, and run many times faster than real-time while being fully decoupled from their respective robots. Thus, the MES can anticipate, saving operational and down time.

\section{B. Optimization-motivated data farming}

At the heart of providing a knowledge base to stakeholders of robots is the discovery and combination of meaningful information in simulation data (see Fig. 6). DGS generate these data as functions of inputs. Such inputs are sets of sensor data along with synthesized joint trajectories having differently parameterized profiles that, however, lead to the same final endeffector pose or posture, depending on the value creation goal. For instance, a polynomial joint trajectory of a degree greater than five has more coefficients than necessary to meet initial and final position, velocity, and acceleration conditions. By varying the extra (i.e., redundant) coefficients $c_{r}$, DGS grow 


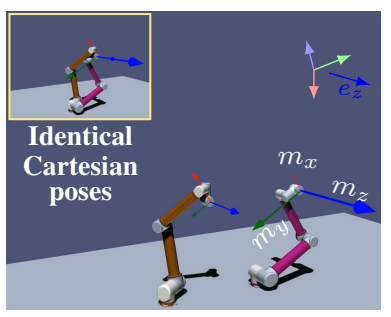

(a) $m_{z}$ (1.h.s) $<m_{z}$ (r.h.s)

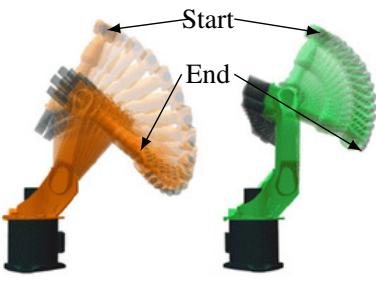

(b) Energy (1.h.s) > Energy (r.h.s)

Fig. 6: l.h.s: The effective mass $m_{z}$ (arrow length) depends on the posture for the same vdRDT, Cartesian pose, and direction. r.h.s: Identical vdRDTs with different energy consumptions depending on joint motions for same start and end postures.

data. To minimize the safety-, usability-, and dexterity-crucial effective mass $m_{z}=\frac{1}{e_{z}^{T} \Lambda_{v}^{-1} e_{z}}$, as stressed in [29], which is felt in Fig. 6(a) at the end-effector in the local Cartesian $e_{z}$-direction, the final posture, optimized in $\eta$, varies for the same end-effector pose. Herein, the submatrix $\Lambda_{v}^{-1} \in \mathbb{R}^{3 \times 3}$ of $\Lambda^{-1}=J M^{-1} J^{T}$ is given in [29] and references therein.

DGS nurture value creation by adjusting input parameters (e.g., redundant coefficients of joint trajectories, time scaling factors of trajectories, compliance parameters, etc.) to cultivate patterns of optimization in data stemming from different generated behaviors of the vdRDT. To this end, DGS populate the space of possible input parameters with particles. DGS update particle positions, similarly to PSO [26], with the aim of inducing a swarm distribution for which the position of at least one particle optimizes cost-functions that generate value.

In Fig. 6(b), for instance, the particle associated with the industrial, green-colored vdRDT on the r.h.s leads to joint trajectories for which the energy consumption is considerably lower than in the case of the identical, orange-colored vdRDT on the 1.h.s. This implies that operational costs are minimized, cash outflow is reduced, and the gross margin is increased.

\section{Increasing relevance in farmed data for focused analysis}

Selection and update of input parameters take advantage of exploration and exploitation skills of the swarm [30], as well as robot performances. For instance, DGS consider only particle positions that give rise to a jerk $\dddot{q}(t)$ lower than a threshold. This reduces drive train wear and vibrations, and positively impacts tracking accuracy and thus manufacturing quality. Furthermore, DGS harness inherent robot properties to focalize optimization. To minimize attitude changes of a free-floating base on which a vdRDT is mounted to service a satellite, as in Fig 7, DGS first compute a current dynamics-dependent basis $\eta_{Z}^{b}=\left\{b_{1}, \cdots, b_{n-3}\right\}$ of the nullspace of $Z\left(m_{i}, I_{i}\right) \in \mathbb{R}^{3 \times n}$ that maps $\dot{q}$ to the base angular velocity $w_{b}=Z \dot{q}$ [31]. $b_{j, j \in\{1, \cdots, n-3\}}$ are basis vectors. Then, DGS farm $\dot{q}\left(t, c_{r}\right)=$ $\alpha_{1}\left(t, c_{r_{1}}\right) b_{1}+\cdots+\alpha_{n-3}\left(t, c_{r_{n-3}}\right) b_{n-3}, \alpha_{j} \in \mathbb{R}$. Varying redundant scalars $c_{r_{j}}$ leads to different final end-effector poses, but with $w_{b}(t)$ kept to the zero vector of $\mathbb{R}^{3}$. Finally, DAS optimize $c_{r_{j}}$ using PSO to steer the end-effector to a desired pose [32] (see Fig. 7(b)). Thus, DGS achieve constrained data growing that increases the information relevance in data, and allows

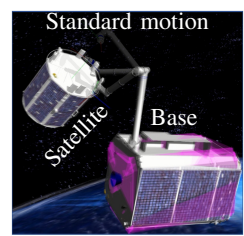

(a) $\Omega\left(t_{i}\right) \neq \Omega\left(t_{f}\right)$.

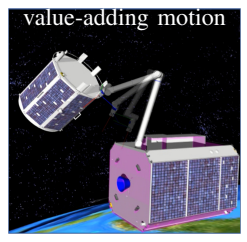

(b) $\Omega\left(t_{i}\right) \approx \Omega\left(t_{f}\right)$.

$$
\begin{gathered}
\Omega=\text { Base attitude } \\
t_{i}=\text { Initial time } \\
t_{f}=\text { Final time }
\end{gathered}
$$

(c) Legend
Fig. 7: vdRDT motions with different base attitude reactions.

DAS to quickly discover meaningful and actionable motions. In contrast to brute-force farming, this also eases the contextualization and interpretation of values being created. In fact, the base control does not use energy-consuming thrusters.

\section{Communication and synchronization}

Timely available desired joint motions of the vdRDT are obtained from the simulation side in Fig. 4 through a three-step approach. Firstly, memory allocations are carried out outside program parts constrained by a bounded and fast response time whereas the computation duration is minimized in each simulation component by exploiting e.g. the sparsity of $J_{m c}$ in section IV-A2. Secondly, asynchronous message passing based on subscribe/publish mechanisms informs the application control when datagrams, in which it is interested, arrive. This decoupling obviates active polling, reduces latency, and fosters scalability. Thirdly, time-critical components are spread among high-priority threads with own schedulers and simulation databases synchronized through the identification of state differences and consistency recovery, as detailed in [5]. Hence, components are concurrently executed at customizable rates while preserving their predefined logical interdependencies. The simulation part with real-time requirements (e.g., control of the vdRDT, transfer of its compliant motions to the robot) is conducted on a PC operated by the real-time system QNX.

In Fig. 4, high-priority DTS receive and send UDP packets to the KUKA LWR 4+ robot running in "command mode" (i.e., cyclic timing and real-time conditions are met) via the Fast Research Interface (FRI). A packet loss is detected through the reflection of counted packets on both the robot and simulation sides [33]. Whereas the robot controller extrapolates joint positions to handle a packet loss [33], a proportional derivative (PD) controller is used on the simulation side to synchronize both robot and vdRDT in terms of tracking accuracy. Specifically, the output of the PD controller, which filters the discrepancy between previous joint positions of the robot and its vdRDT, is added to the current position vector of the vdRDT to obtain desired joint positions sent to the robot.

When the bridged communication between PC for realtime simulation and, e.g., the master controller of an EtherCat capable industrial robot, devices like EK1100 (bus coupler), EL1809/EL2809 (I/Os for gripper control), as well as an incremental encoder interface for controlling the linear axis is conducted over EtherCat, distributed clock synchronization of the EtherCat bus allows for compensating transmission delays and clock drifts. In this case, communicating system modules are sharing the same system time with an accuracy in $\mu s$. 


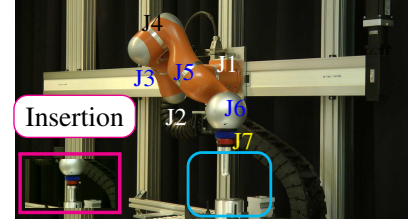

(a) Setup of the 7 DoF robot.

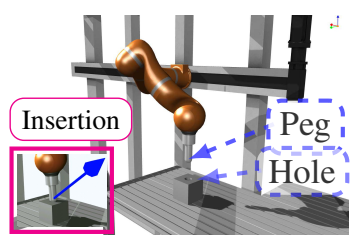

(b) Virtual setup with the vdRDT.
Fig. 8: Peg-in-hole experiment to compare joint torques.

\section{Applications}

The effectiveness of vdRDTs was evaluated in many applications. Acting as informative robot surrogates, they extended user and robot capacities and met expectations on RDTs. Smallest real-time simulation timestep was $1[\mathrm{~ms}]$. PCs, 64 Bit i7 multi-core CPU at up to $4.1[\mathrm{Ghz}], 18[\mathrm{~GB}]$ RAM, were used.

\section{A. Validation of dynamics capture}

The goal of the experiment was to compare the dynamics of the robot and its vdRDT during free-space motions and physical interactions. The robot in Fig. 8(a) was controlled to vertically insert a peg attached to its end-effector into a hole. The robot moved the peg toward the hole in the time intervals $t \in[0, \cdots, 20]_{[s]}$ and $[40, \cdots, 60]_{[s]}$. Real external torques resulting from wrenches exerted on the robot during insertion maneuvers for $t \in[20, \cdots, 40]_{[s]}$ and $[60, \cdots, 70]_{[s]}$ were sent to the vdRDT as UDP datagrams. For that, the KUKA FRI was used. The robot and its digital sibling were fed with the same desired joint trajectories, and operated under joint admittance control with identical joint stiffness values.

Forms and amplitudes of joint torques of the robot and its vdRDT matched during free-space and physically constrained motions, as shown in Fig. 9 and emphasized by the highest discrepancy for each joint given in Table I. Notice that these maximal values occurred within a very short time interval during physical interactions with the hole. To further investigate this observation, an index for the spread of simulated joint torques
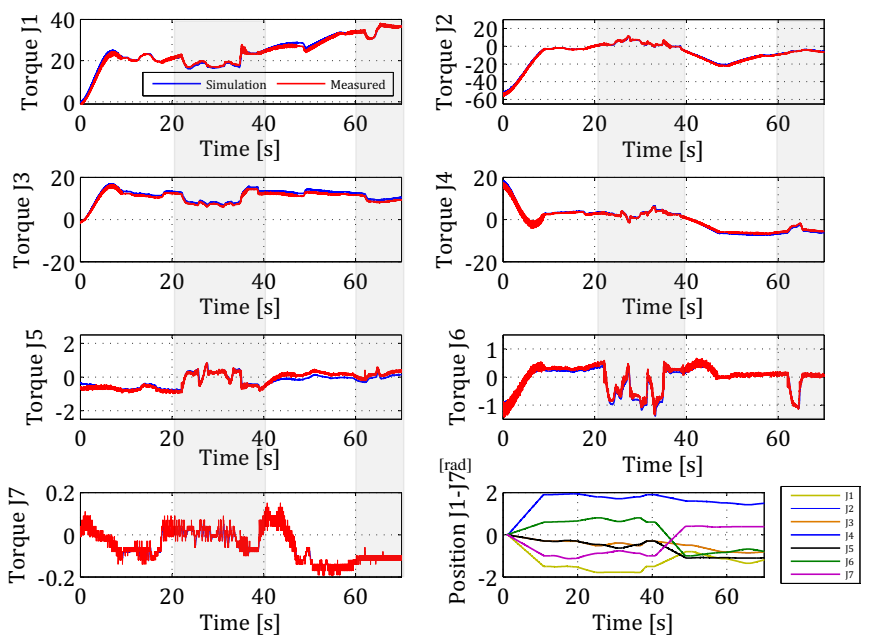

Fig. 9: Real and virtual joint torques [Nm] during free space and physically constrained robot and vdRDT motions in Fig. 8.

\begin{tabular}{|c|c|c|c|c|c|c|c|}
\hline Joints & Joint1 & Joint2 & Joint3 & Joint4 & Joint5 & Joint6 & Joint7 \\
\hline \hline$E_{\tau_{i}}[\mathrm{Nm}]$ & 4.22 & 4.32 & 2.33 & 3.66 & 0.71 & 0.73 & 0.09 \\
\hline
\end{tabular}

TABLE I: Absolute value $E_{\tau_{i}}$ of the maximum discrepancy between measured and simulated joint torques in Fig. 9.

$\tau_{J_{s}}(k)$ about measured joint torques $\tau_{J_{m}}(k)$, at discrete time steps $k$, was computed and analyzed. Collected joint torques were decomposed in eight pairs of different sets of simulated and measured values for each of the seven joints. Each pair had $N_{m}=4375$ values for measured and $N_{m}=4375$ values for simulated joint torques for each joint. The root mean square error was then computed for the $i$-th joint (see Fig. 8(a)) as $R_{i}=\sqrt{\sum_{k=1}^{N_{m}} \frac{\left(\tau_{J_{s_{i}}}(k)-\tau_{J_{m_{i}}}(k)\right)^{2}}{N_{m}}}$. Figure 10 indicates that $R_{i} \leq 1.52[\mathrm{Nm}]$ for all joints, which is approximately 2.82 times smaller than the maximal value in Table I. Apart of motions in Figs. 10 (a) and (f) characterized by high $\dot{q}$ and $\ddot{q}$, motions related to Figs. 10 (b), (c), (d), (e), (g), and (h) reveal that $R_{i, i \in\{1, . ., 7\}}<1.13[\mathrm{Nm}]$. This accurate dynamics capture was achieved for both large (e.g., 1. and 2. joint) and small (e.g., 3. and 5. joint) joint motions. Immediately before and after insertions, e.g. $t \in[19.5, \cdots, 19.99]_{[s]}$ and $t \in[39, \cdots, 40]_{[s]}$, the robot was almost at rest, showing that measured and simulated gravity torques were also similar. Not only the sum, but also single contributions (e.g., inertia- and velocity-dependent) that sum up in equation (2a) were captured. Associated matrices and vectors involved in these contributions, as defined in equations (2a)-(2c), offer a library for developing model-based algorithms for customized vdRDTs.

\section{B. Rendering the desired compliance of joints}

The reaction of admittance-controlled joints of a two DoF vdRDT to external joint torques was evaluated. Synthesized external joint torques are given in Fig. 11(c). Targeted stiffness and damping values of both joints are depicted in Fig. 11(d). Desired and compliant joint trajectories are shown
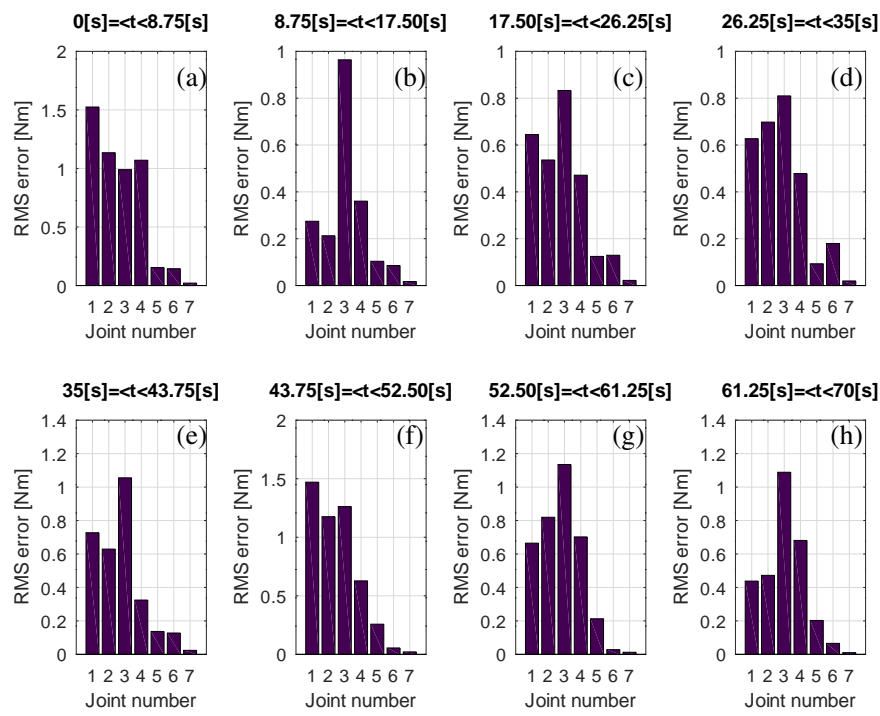

Fig. 10: The root mean square (RMS) error $R_{i}$ related to the difference between joint torques of the robot and its vdRDT. 


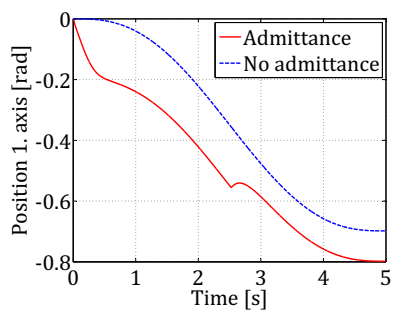

(a) Position of the 1. joint.

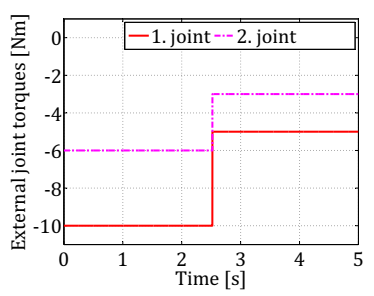

(c) External joint torques.

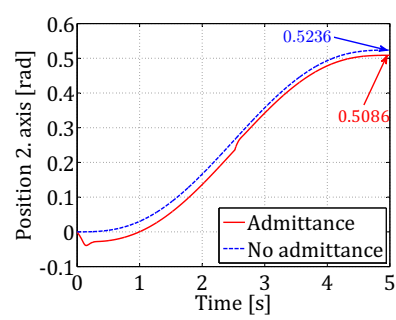

(b) Position of the 2. joint.

\begin{tabular}{|c|c|c|}
\hline Param. .Joints & Joint1 & Joint2 \\
\hline \hline Inertia $\left[\mathrm{s}^{2} \mathrm{Nm} / \mathrm{rad}\right]$ & 1 & 1 \\
\hline Damping $[\mathrm{sNm} / \mathrm{rad}]$ & 14.14 & 28.28 \\
\hline Stiffness $[\mathrm{Nm} / \mathrm{rad}]$ & 50 & 200 \\
\hline
\end{tabular}

(d) Admittance parameters (Param.).

Fig. 11: Verification of joint admittance control.

in Figs. 11(a) and 11(b). They indicate that the transient phase $(t \approx 2.5[s])$ was overshoot-free and the desired stiffness values were accurately rendered for both joints at the steady state.

\section{C. vdRDT-based manual guidance of a stiff robot}

The position-controlled robot in Fig. 12 was made compliant by using its admittance-controlled vdRDT. Whereas the vdRDT was fed with external joint torques that stemmed from physical interactions between an operator and the robot, compliant joint trajectories of the vdRDT were sent back to and tracked by the robot as desired motion. On this basis, the operator manually guided the otherwise stiff robot to a desired posture. Advantages of freely moving the robot include simplified task programming and flexible payload transport. To raise safety awareness, the vdRDT enriched the manual guidance with visual cues displaying information from the captured dynamics in real-time upon request. These encompassed inertial properties of the robot. Operators were thereby capable of easily planning end-effector poses for which the mass respectively

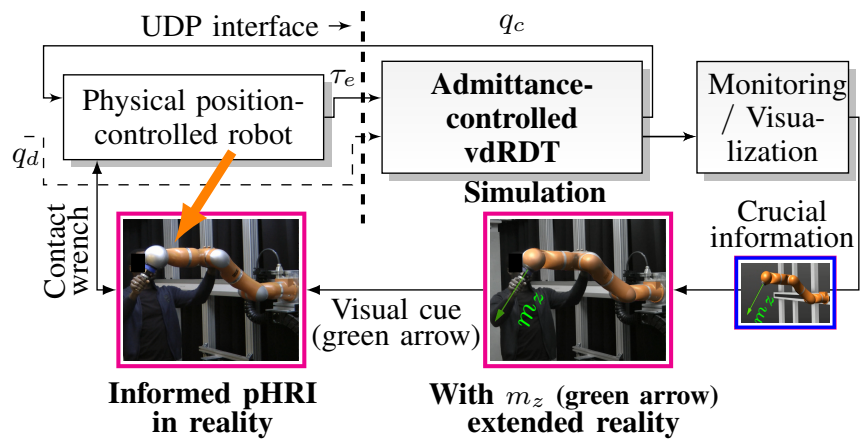

Fig. 12: The position-controlled robot is manually guided by using its vdRDT, which enriches the guidance by delivering the effective mass (green arrow). $q_{d}, q_{c}$, and $\tau_{e}$ are desired joint positions, compliant positions, and sensed external torques.

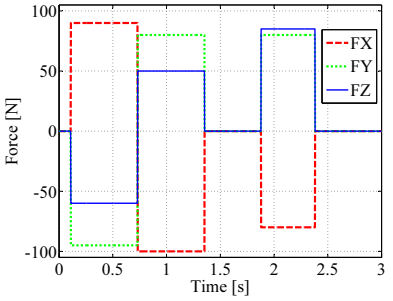

(a) Forces acting at TCP.

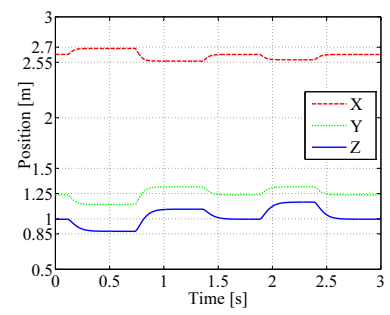

(c) Compliant TCP position.
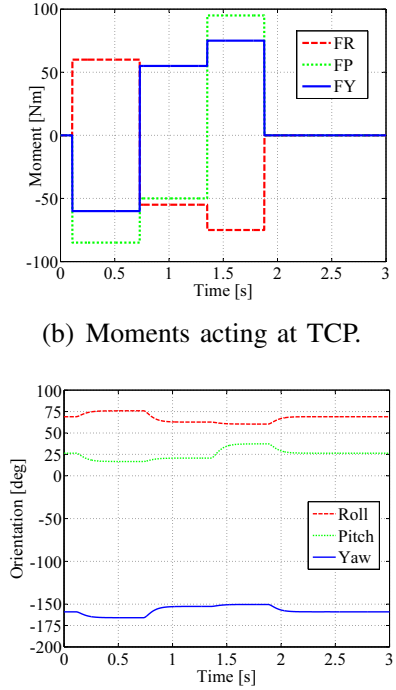

(d) Compliant TCP orientation. (b) Moments acting at TCP.

Fig. 13: Impedance control with parameters from TABLE II.

(resp.) inertia felt along resp. around a given direction is minimized. Also, we observed that appropriately choosing joint damping resp. inertia values canceled possible vibrations felt resp. enhanced the guidance experience made by operators.

\section{Rendering the desired Cartesian end-effector compliance}

The end-effector of the vdRDT in Fig. 8(b) was subject to external wrenches shown in Figs. 13(a) and 13(b). Both the sign and amplitude of external disturbances abruptly changed. Before physical interactions, the Cartesian position and orientation of the end-effector were $(2.621,1.232,0.992)_{[\mathrm{m}]}$ and $(68.752,26.355,-158.703)_{[\mathrm{deg}]}$ at rest. During physical interactions, Figs. 13(c) and 13(d) show that the vdRDT accommodated the varying disturbances according to the aperiodic compliance model given by Table II without overshoot. Notice also that targeted stiffness values were accurately reached for the six position and orientation dimensions. Figures 13(c) and 13(d) reveal for $t \in[0.75,1]_{[s]}$ how the end-effector settles, and thus capture how the compliance model impacts not only safety, but also cycle time and, thereby, energy consumption.

\section{E. vdRDT-based human-robot cooperation in manufacturing}

The stiff behavior of the end-effector of the positioncontrolled robot in Fig. 14(a) was made compliant by tracking the force-sensitive behavior of its vdRDT\#1 in Fig. 14(b). To apply pressure, remove the workpiece, and place it back on the table, as in pHRI-based manufacturing, the vdRDT was fed with desired Cartesian motions and external wrenches sensed

\begin{tabular}{|c|c|c|c|}
\hline Pose $\backslash$ Parameters & Inertia & Damping & Stiffness \\
\hline \hline$X$ & $1\left[s^{2} \mathrm{~N} / \mathrm{m}\right]$ & $77.45[\mathrm{sN} / \mathrm{m}]$ & $1500[\mathrm{~N} / \mathrm{m}]$ \\
\hline $\mathrm{Y}$ & $1\left[s^{2} \mathrm{~N} / \mathrm{m}\right]$ & $63.24[\mathrm{sN} / \mathrm{m}]$ & $1000[\mathrm{~N} / \mathrm{m}]$ \\
\hline $\mathrm{Z}$ & $1\left[s^{2} \mathrm{~N} / \mathrm{m}\right]$ & $44.72[\mathrm{sN} / \mathrm{m}]$ & $500[\mathrm{~N} / \mathrm{m}]$ \\
\hline Roll, Pitch, Yaw & $1\left[s^{2} \mathrm{Nm} / \mathrm{rad}\right]$ & $44.72[\mathrm{sNm} / \mathrm{rad}]$ & $500[\mathrm{Nm} / \mathrm{rad}]$ \\
\hline
\end{tabular}

TABLE II: Parameters for Cartesian impedance verification. 

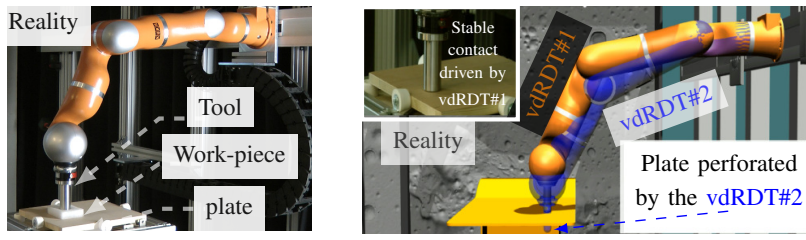

(a) The phyical robot is inter- (b) Compliant (orange) and stiff (blue) acting with the workpiece. vdRDTs in interaction with the table.

Fig. 14: vdRDT-based Cartesian guidance of the robot.

at the end-effector. Compliant joint motions of the impedancecontrolled vdRDT were sent back in real-time to the robot, as in Fig. 12, which thereby accommodated wrenches. As shown in Figs. 15 (A,B), the polystyrene workpiece was clamped after a downward motion of the end-effector. The latter maintained task-specific forces on the workpiece according to the desired stiffness and steady state assigned to the vdRDT. Figs. 15 $(\mathrm{C}, \mathrm{D})$ and $(\mathrm{F}, \mathrm{G}, \mathrm{H})$ depict how the operator easily grasped and moved the end-effector upward, removed the workpiece, and put it back. Both Figs. 15 (E) and (I) show how the robot safely interacts with objects having different materials.

A vdRDT not only augments and extends capabilities of the robot, but also carries out investigative tasks for which the usage of the robot is prohibitive. This is the case for the study illustrated in Fig. 14(b). The vdRDT\#2 identified critical stiffness values for which the end-effector pierced the workpiece or/and plate for a given final Cartesian pose. Such values help prevent damages when planning robot tasks.

\section{F. vdRDT-based monitoring of industrial robots and processes}

In experiments depicted in Fig. 16, which were conducted in the manufacturing-related projects ReconCell resp. ProDemo-2, vdRDTs in Fig. 16(b) and Fig. 16(d) mirrored the current motions of their associated robots in Figs. 16(a) and 16(c). The tight positional coupling, which is typical of RDTs (see section III), allowed for monitoring the posture as well as spatial vicinity between robots and obstacles to detect possible collisions. Obstacles either physically existed, or were virtually added to prospectively extend the robot workspace for what-if analysis. To detect imminent collisions, ensure safety as stated in Fig 3, vdRDTs as well as static and moving obstacles were

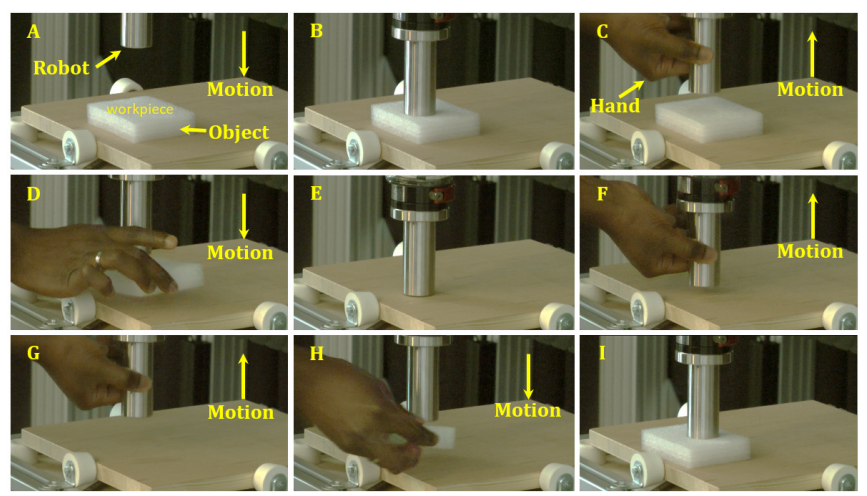

Fig. 15: Cartesian vdRDT-based manual guidance of a robot.

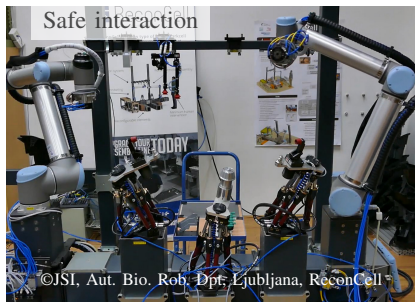

(a) Real setup.

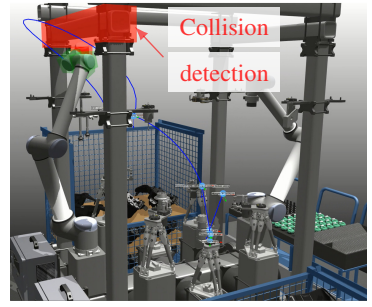

(b) Collision detection.

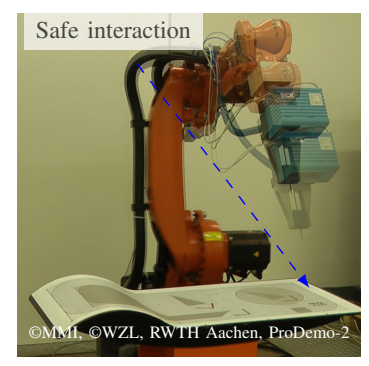

(c) Real setup.

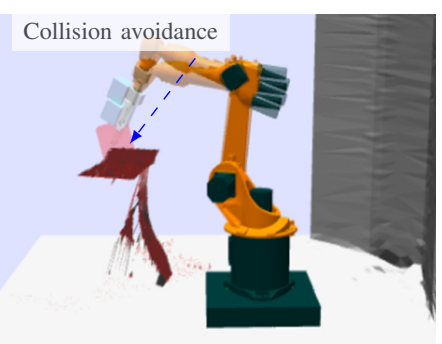

(d) Collision avoidance.
Fig. 16: Tightly coupled vdRDTs for collision handling.

enveloped with convex safety hulls, as shown in Fig. 16(b). The geometry of obstacles was also accurately approximated beforehand as 3D point cloud (see Fig. 16(d)) based on the superposition of outputs of the 2D laser scanner mounted on the end-effector in Fig. 16(c). Sets of joint positions associated with an incremental rotation of the end-effector of the vdRDT were forwarded to the robot control unit as desired scanning posture. Then, scans were triggered in-between from the simulation. Interpenetrations between bounding hulls of vdRDTs and obstacles were detected, and Cartesian information on involved links and obstacles were used to avoid collisions.

\section{G. vdRDT-based reconfiguration of satellites}

Mature vdRDTs were used to alleviate the complexity and costs incurred by a new satellite launch. In other words, vdRDTs helped study the feasibility of robotized satellite upgrades in virtual space-like testbeds within the scope of the project IBOSS-3 (see the r.h.s of Fig. 17(b)). Such an adaptation was based upon the reconfiguration of satellite building blocks to meet specific mission requirements. Two robots were

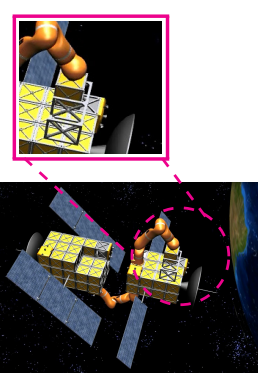

(a) Block insertion.

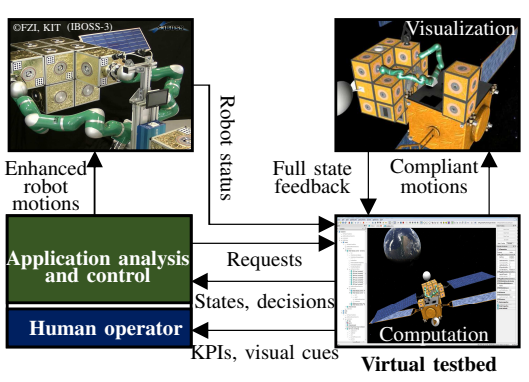

(b) Assessing robotized satellite reconfiguration.
Fig. 17: vdRDT-based assessment of robotized satellite reconfigurations conducted in cyber-physical applications. 
mounted on a fixed base to assess manipulations necessary for repositioning tasks (see upper 1.h.s of Fig. 17(b)). Whereas one robot fixed the satellite, the other relocated its blocks. Desired joint motions of robots were used as inputs for a simultaneous completion of the same relocation tasks in the testbed. Two vdRDTs resp. associated with one of both robots were mounted on a free-floating base (see upper r.h.s of Fig. 17(b)). Each vdRDT tracked desired motions of its physical sibling to interact (i.e., service) in real-time with a free-floating satellite. Both robots and their vdRDTs were operated under joint admittance control with identical desired stiffnesses.

In the phase of satellite approach, paths taken by each vdRDT led to modifications of the relative attitude between the base and satellite. This induced positional mismatches as well as unplanned interaction wrenches between vdRDTs and the satellite with finite stiffness. Such disturbances did not occur in the physical setup, since the base and satellite were fixed (upper 1.h.s of Fig. 17(b)). Hence, vdRDTs helped uncover limitations of standard motion planning schemes that can jeopardize the satellite reconfiguration in space. By contrast, vdRDTs returned motions that accommodated external wrenches to the application control units of robots in real-time.

Once the satellite was captured, vdRDTs also sent back 6D poses of grasped building blocks and contact wrenches, as well as performance indexes, to the robot application for performance analysis by using DTS. For instance, vdRDTs estimated, visualized, and minimized the effective mass felt by blocks at their end-effectors for safe grasping and inserting maneuvers (see Fig. 17(a)). Joint trajectories that minimize the energy consumption and predictions on energy regeneration capabilities of robots were returned by vdRDTs to application control units for the design of cost-efficient robot motions.

\section{DISCUSSION}

Developing vdRDT is a nascent research field full of challenges and opportunities. Although a vdRDT is a source of operational efficiency in non-adjacent industrial sectors, its impact further increases with the accuracy of the dynamics capture. Hence, using e.g. deep learning to consider intricate effects unmodeled so far like temperature-induced deformations and disturbances, such as power cords in Fig. 16(c), deserves additional attention, depending on the required precision. In either domain, however, the vdRDT demonstrates that it extends skills and augments performances of robots and capacities of stakeholders at different levels of decision making. Personalized insights and situational awareness that engages people through immersive visual cues make the paired robot virtually appealing and accessible as vdRDT to both experts and novices. This vdRDT-Human-Symbiosis shifts the vdRDT utility from purely technical and economic goals, to educational and societal knowledge transfer, opening up new avenues. In fact, since a vdRDT is intrinsically safe and scalable by design without engendering additional costs, it can accelerate the penetration and adaptation of advanced robotics to specific needs in developing countries. Early promotions in schools, vocational training, and public outreach fueled by
vdRDTs will become reality. Such a pervasiveness of vdRDTs helps one swiftly understand how the latest and locally adapted robotized solutions tackle issues faced in prioritized domains and fosters self-determination. This will be done long before financial and physical resources are committed. When enriched with measurements local to different countries, interconnected (e.g., via tactile internet) vdRDTs will share actionable insights for fruitful collaborations with prolific labs around the globe.

\section{CONCLUSION}

The vdRDT is evolving and allows for building a knowledge asset as a competitive edge in current and future robotized cyber-physical applications. The presented work contributes to this goal by developing mature vdRDTs that farm and harness data. On this basis, different vdRDTs are used to identify nonnominal advantages that drive challenging robotized endeavors. Obtained results indicate that vdRDTs minimize operational efforts and costs, increase visibility and agility, and energize cross-disciplinary collaborations with personalized insights in applications ranging from manufacturing to space servicing. Robot stakeholders can take advantage of vdRDTs to achieve digital proficiency and beneficial business outcomes.

\section{REFERENCES}

[1] H. Aaron, "Covid-19 brings increased visibility to the role of robotics," https://www.automationworld.com/factory/robotics/article/21130808/ covid19-brings-increased-visibility-to-the-role-of-robotics, Automation world, Apr. 2020, last accessed: 08 June 2020.

[2] B. Wire, "Covid-19 impact on the global industrial robotics market," https://www.businesswire.com/news/home/20200424005397/en/ COVID-19-Impact-Global-Industrial-Robotics-Market--, Business Wire, Apr. 2020, last accessed: 08 June 2020.

[3] A. A. Malik and A. Bilberg, "Digital twins of human robot collaboration in a production setting," Procedia manufacturing, vol. 17, pp. 278-285, 2018.

[4] K. Nilsson and R. Johansson, "Integrated architecture for industrial robot programming and control," Robotics and Autonomous Systems, vol. 29, no. 4, pp. 205-226, 1999.

[5] A. Wahl, R. Waspe, M. Schluse, and J. Roßmann, "Parallel simulation of virtual testbed applications," in International Conference on Simulation and Modeling Methodologies, Technologies and Applications. Springer, 2017, pp. 177-199.

[6] A. Overn, "Industry 4.0 - digital twins and opc ua," in Master of Science in Mechanical Engineering. NTNU - Norwegian University of Science and Technology, 2018.

[7] A. Bilberg and A. A. Malik, "Digital twin driven human-robot collaborative assembly," CIRP Annals, vol. 68, no. 1, pp. 499-502, 2019.

[8] V. Havard, B. Jeanne, M. Lacomblez, and D. Baudry, "Digital twin and virtual reality: a co-simulation environment for design and assessment of industrial workstations," Production \& Manufacturing Research, vol. 7, no. 1, pp. 472-489, 2019

[9] H. Laaki, Y. Miche, and K. Tammi, "Prototyping a digital twin for real time remote control over mobile networks: Application of remote surgery," IEEE Access, vol. 7, pp. 20325-20336, 2019.

[10] R. Rocca, P. Rosa, C. Sassanelli, L. Fumagalli, and S. Terzi, "Integrating virtual reality and digital twin in circular economy practices: A laboratory application case," Sustainability, vol. 12, no. 6, p. 2286, 2020.

[11] F. Lima, C. N. de Carvalho, M. B. Acardi, E. G. dos Santos, G. B. de Miranda, R. F. Maia, and A. A. Massote, "Digital manufacturing tools in the simulation of collaborative robots: towards industry 4.0," Brazilian Journal of Operations \& Production Management, vol. 16, no. 2, pp. 261-280, 2019.

[12] A. Ghosh, D. Alonso Paredes Soto, S. M. Veres, and J. Rossiter, "Human robot interaction for future remote manipulations in industry 4.0," in IFAC-PapersOnLine. 21st IFAC World Congress 2020, 12-17 Jul 2020, Berlin, Germany. International Federation of Automatic Control (IFAC), Accepted Version, 2020, pp. 2-9, http://eprints.whiterose.ac.uk/157791/ 1/IFAC20_4304_MS.pdf. 
[13] N. Yamanobe, H. Fujii, T. Arai, and R. Ueda, "Motion planning by integration of multiple policies for complex assembly tasks," Cutting Edge Robotics 2010, p. 81, 2010

[14] B. Li, G. Li, W. Lin, and P. Xu, "Design and constant force control of a parallel polishing machine," in 2014 4th IEEE International Conference on Information Science and Technology. IEEE, 2014, pp. 324-328.

[15] W. Kraus, "Force control of cable-driven parallel robots," Ph.D. dissertation, Stuttgart University, 2016.

[16] A. Ajoudani, N. G. Tsagarakis, and A. Bicchi, "On the role of robot configuration in cartesian stiffness control," in 2015 IEEE International Conference on Robotics and Automation (ICRA). IEEE, 2015, pp. 1010-1016.

[17] A. M. Madni, C. C. Madni, and S. D. Lucero, "Leveraging digital twin technology in model-based systems engineering," Multidisciplinary Digital Publishing Institute, Systems, vol. 7, no. 1, p. 7, 2019.

[18] T. Jung, "Methoden der mehrkörperdynamiksimulation als grundlage realitätsnaher virtueller welten," Ph.D. dissertation, RheinischWestfälische Technische Hochschule Aachen, RWTH Aachen University, 2011.

[19] K. Erleben, "Velocity-based shock propagation for multibody dynamics animation," ACM Transactions on Graphics (TOG), vol. 26, no. 2, pp. 12-es, 2007.

[20] S. Haddadin, A. De Luca, and A. Albu-Schäffer, "Robot collisions: A survey on detection, isolation, and identification," IEEE Transactions on Robotics, vol. 33, no. 6, pp. 1292-1312, 2017.

[21] M. Bjerkeng and K. Y. Pettersen, "A new coriolis matrix factorization," in 2012 IEEE International Conference on Robotics and Automation. IEEE, 2012, pp. 4974-4979.

[22] F. Caccavale, C. Natale, B. Siciliano, and L. Villani, "Six-dof impedance control based on angle/axis representations," IEEE Transactions on Robotics and Automation, vol. 15, no. 2, pp. 289-300, 1999.

[23] X. Zhang, W. Yang, X. Cheng, and Y. Chen, "Stiffness identification for serial robot manipulator based on uncertainty approach," in International Conference on Intelligent Robotics and Applications. Springer, 2011 , pp. 378-388.
[24] C. Massimo, "Notes on the kuka lwr4 dynamic model." http: //coppeliarobotics.com/files/contributions/LBR4p_dynamic_model.pdf, 2014.

[25] C. Gaz, F. Flacco, and A. De Luca, "Extracting feasible robot parameters from dynamic coefficients using nonlinear optimization methods," in 2016 IEEE international conference on robotics and automation (ICRA). IEEE, 2016, pp. 2075-2081.

[26] M. Clerc, Particle swarm optimization. John Wiley \& Sons, Online ISBN: 9780470612163, Print ISBN: 9781905209040, 2010.

[27] V. Roberge and M. Tarbouchi, "Parallel particle swarm optimization on graphical processing unit for pose estimation," WSEAS Trans. Comput, vol. 11 , no. 6 , pp. 170-179, 2012.

[28] A. Dietrich, C. Ott, and A. Albu-Schäffer, "An overview of null space projections for redundant, torque-controlled robots," The International Journal of Robotics Research, vol. 34, no. 11, pp. 1385-1400, 2015.

[29] J. G. Petersen, S. A. Bowyer, and F. R. y Baena, "Mass and friction optimization for natural motion in hands-on robotic surgery," IEEE Transactions on Robotics, vol. 32, no. 1, pp. 201-213, 2016.

[30] K. E. Parsopoulos and M. N. Vrahatis, "Parameter selection and adaptation in unified particle swarm optimization," Mathematical and Computer Modelling, vol. 46, no. 1-2, pp. 198-213, 2007.

[31] D. N. Nenchev, "Reaction null space of a multibody system with applications in robotics," Mechanical Sciences, vol. 4, no. 1, pp. 97112,2013

[32] E. Guiffo Kaigom, T. J. Jung, and J. Roßmann, "Optimal motion planning of a space robot with base disturbance minimization," in 11th Symposium on Advanced Space Technologies in Robotics and Automation, ASTRA, European Space Agency, ESA, Noordwijk, Nederland, 2011, pp. 1-6.

[33] G. Schreiber, A. Stemmer, and R. Bischoff, "The fast research interface for the kuka lightweight robot," in IEEE Workshop on Innovative Robot Control Architectures for Demanding (Research) Applications How to Modify and Enhance Commercial Controllers (ICRA 2010). Citeseer, 2010, pp. 15-21. 